\title{
ÓBITO POR NEGLIGÊNCIA NO TRATAMENTO DE DERMATITE ATÓPICA:
} RELATO DE CASO

\author{
Maristela dos Santos Vieira ${ }^{1}$; Estela Vieira de Souza Silva ${ }^{2}$; Regiani Nascimento \\ Gagno Pôrto ${ }^{3}$; Moema Pacheco Chediak Matos ${ }^{3}$, Ana Paula Iglesias Santin ${ }^{3}$ \\ ${ }^{1}$ Acadêmica em Medicina Veterinária, da Escola de Veterinária e Zootecnia da \\ Universidade Federal de Goiás (EVZ/UFG), Goiânia, Brasil \\ (maristela.santosv@gmail.com) \\ ${ }^{2}$ Pós-graduanda do Programa de Mestrado em Ciência Animal da EVZ/UFG, \\ Goiânia, Brasil. \\ ${ }^{3}$ Professor Associado da EVZ/UFG, Goiânia, Brasil (regianiporto@hotmail.com; \\ mpcmatos@ufg.br; apisantin@gmail.com)
}

Recebido em: 06/04/2019 - Aprovado em: 10/06/2019 - Publicado em: 30/06/2019 DOI: 10.18677/EnciBio_2019A93

\begin{abstract}
RESUMO
Um cão, macho, SRD, adulto e portador de dermatite atópica canina, que devido à ausência da terapia de suporte e sem atendimento médico veterinário, teve seu estado bastante agravado. Ao ter sido resgatado da tutora, por uma protetora de animais, para tratamento adequado, não resistiu e veio a óbito. $O$ animal foi encaminhado para a realização do exame anatomopatológico, durante a necroscopia observou-se múltiplas ulceras cutâneas, eritrema, presença de tricobezoar estomacal, múltiplas ulceras estomacais e intestinais associadas à gastrorragia e enterorragia. Ao exame microscópico identificou-se pneumonia intersticial crônico ativa e glomerulonefrite. A causa mortis foi por choque hipovolêmico decorrente da gastrorragia e enterorragia crônicas, ocasionadas pela presença de um piloconcremento que levou à formação das úlceras gastrointestinais. Portanto, foi possível evidenciar a negligência da tutora, permitindo que a doença evoluísse, sem encaminhar o animal ao médico veterinário para os devidos cuidados, sendo a negação do atendimento médico veterinário considerada maus tratos, de acordo como o decreto lei no 24.645, de junho de 1934 .
\end{abstract}

PALAVRAS-CHAVE: Cão, DAC, Maus-Tratos, Tricobezoar

\section{DEATH AND NEGLIGENT TREATMENT OF ATOPIC DERMATITIS: A CASE REPORT}

\begin{abstract}
Mongrel dog, male, adult, with canine atopic dermatitis and no veterinary medical care. The dog was rescued from its tutor by an animal protector. The animal was referred to appropriate veterinary treatment, but died. Anatomopathological evaluation showed active chronic interstitial pneumonia, glomerulonephritis associated, purulent dermatitis and it has a trichobezoar inside de stomach. The
\end{abstract}


cause of death was hypovolemic shock due to gastrointestinal hemorrhage arising from the presence of trichobezoar. Therefore, it was possible to demonstrate the tutor negligence, which allowed the progression of disease, since animal has no adequate care, the absence of veterinary care is considered ill-treatment, according to the Decree Law No. 24.645, from June 1934.

KEYWORDS: Dog, Atopia, Mistreatment, Trichobezoar

\section{INTRODUÇÃO}

O abandono é um tipo de maus tratos comumente observado nas cidades brasileiras. Entretanto, apesar desta atitude ser uma prática comum, são escassas as pesquisas acerca do tema, bem como sobre a adoção de animais (MENDONÇA ; MELO, 2014). Estudos evidenciaram que quanto maior for o grau de instrução dos tutores, mais conhecimento estes têm sobre as responsabilidades $e$ as consequências da adoção ou aquisição de um animal de estimação (DOMINGUES et al., 2015).

Baquero et al., (2015) concluíram, em sua pesquisa, que a presença de algum tipo de enfermidade é uma das principais razões que levam ao abandono de animais de estimação, como cães e gatos, outros fatores como agitação, tamanho e problemas de comportamento também são citados como possíveis motivadores do abandono. Entretanto, os relatos sobre os reais motivos que levam a esta atitude são reduzidos, uma vez que a pessoa que abandona não costuma fornecer informações sobre o assunto (MENDONÇA; MELO, 2014).

Muitos animais resgatados costumam apresentar problemas dermatológicos, entre estes, destaca-se a dermatite atópica canina (DAC), que é uma doença pruriginosa, inflamatória e de elevada ocorrência em cães, sendo caracterizada por uma reação de hipersensibilidade do tipo I, mediada pela imunoglobulina $E$, e que necessita de tratamento contínuo. Esta enfermidade ocorre em animais geneticamente predispostos a desenvolverem reação de hipersensibilidade a alérgenos do ambiente, que podem penetrar pela pele ou por via oral, pela ingestão dos mesmos (DIOGO et al., 2014; GROSSEL; POVALUK 2016; SANTOS; SANTOS, 2016; MEDEIROS, 2017; ALVES et al., 2018; TIMM et al., 2018).

Os sinais clínicos mais comuns da dermatite atópica incluem prurido, eritema cutâneo, escoriações, alopecia, liquenificação e hiperpigmentação. As regiões mais acometidas são face, pavilhão auricular, região ventral, locais de flexuras e patas. A maioria dos animais desenvolve o problema antes dos três anos de idade (BIZIKOVA et al., 2015; SARIDOMICHELAKISA; OLIVRY, 2016; MEDEIROS, 2017; TIMM et al., 2018).

É imprescindível que haja o acompanhamento do médico veterinário, visto que, é necessário um tratamento multimodal e gestão permanente da doença crônica (PUCHEU-HASTON, 2016). Revisões periódicas das terapias adotadas e a adaptação das mesmas às necessidades do paciente devem ser realizadas, além disso, a manutenção com terapia tópica é citada como método para evitar o reaparecimento dos sinais clínicos da doença (SARIDOMICHELAKISA; OLIVRY 2016).

O prurido gerado por estas dermatites pode levar à lambedura e/ou mordedura, e os animais acabam ingerindo pelos (MACPHAIL, 2002). A ingestão excessiva de pelos e/ou cabelos (tricofagia) é uma condição que pode levar à formação de tricobezoares, sendo estes mais frequentemente encontrados no 
estômago. O tricobezoar costuma causar obstrução total ou parcial do trato gastrointestinal devido à formação de uma massa complexa e endurecida, podem, ainda, estender-se para o intestino, fato denominado de síndrome de Rapunzel (PÉREZ et al., 2014; COUCEIRO et al., 2016; FINALE et al., 2018; KHAN et al., 2018).

Deste modo, a tutoria de animais portadores de dermatite atópica canina requer maior atenção e possivelmente gastos extras com tratamento (NOLI et al., 2011). Assim, ressalta-se a importância da guarda responsável, na qual o tutor está ciente de seus deveres e se responsabiliza em cumprí-los (MENDONÇA; MELO, 2014).

O presente trabalho teve como objetivo relatar um caso de dermatite atópica resultando em formação de tricobezoar gástrico, e com evolução para o óbito devido negligência da tutora.

\section{RELATO DO CASO}

Chegou ao Setor de Patologia Animal da Escola de Medicina Veterinária e Zootecnia da Universidade Federal de Goiás, um cão, macho, adulto, SRD, para realização de exame necroscópico. De acordo com o relato da pessoa que resgatou o animal, este foi retirado da tutora que o havia adotado, para ser submetido a tratamento médico veterinário. Ao exame clínico, o animal encontrava-se debilitado, apático, com extensas lesões cutâneas, sem ter sido submetido a nenhum tratamento prévio (Figura 1). De acordo com histórico anterior do animal, este era portador de dermatite atópica canina, sendo a doença de conhecimento da tutora, desde o momento da adoção, no qual o animal encontrava-se sadio (Figura 2). Mesmo após o encaminhamento ao médico veterinário, em decorrência do estado avançado da doença e dos problemas secundários, o animal veio a óbito.

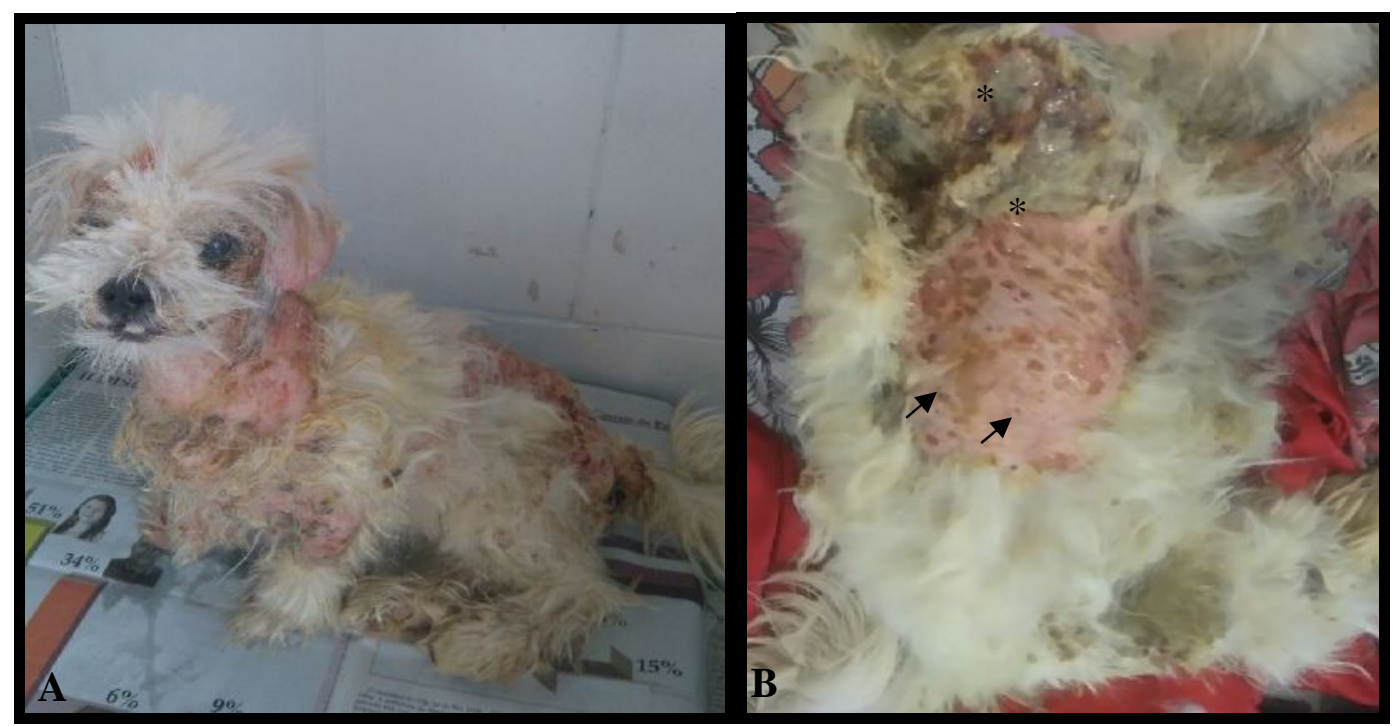

FIGURA 1 Cão, macho, adulto, SRD, portador de DAC. A) Extensas áreas de alopecia, eritema e presença de lesões na pele, de distribuição generalizada. B) Descolamento da pele, na região abdominal lateral direita, apresentando material purulento (asterisco) e múltiplas úlceras de tamanhos variados (setas).

Fonte: Arquivo pessoal (2016). 


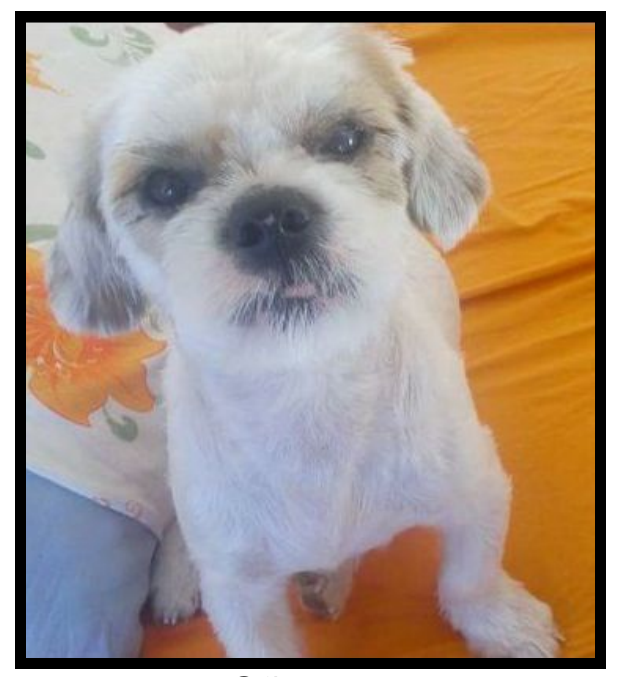
FIGURA 2 Cão, macho, adulto, SRD, portador de DAC, no dia da adoção. Aspecto geral bom, pele e pelagem íntegras.

Fonte: Arquivo pessoal (2016).

Durante o exame anatomopatológico, notou-se extensas áreas de alopecia na cabeça, tronco e membros, sempre associadas à presença de inúmeras úlceras cutâneas de tamanhos variados e áreas eritematosas (Figura 3). A mucosa oral encontrava-se pálida e na pelagem da região perianal observou-se a presença de fezes enegrecidas. À abertura da cavidade torácica verificou-se que o pulmão apresentava áreas de hemorragia. No coração verificou-se discreta dilatação da cavidade direita e aumento de volume da musculatura do ventrículo esquerdo.

$\mathrm{Na}$ cavidade abdominal observou-se acentuada e generalizada palidez dos órgãos. O fígado apresentava evidenciação do padrão lobular. À abertura do estômago foi retirado tricobezoar, de aproximadamente $11 \mathrm{~cm}$ de largura por $6 \mathrm{~cm}$ de altura (Figura 3), que ocupava a totalidade da luz do órgão. Após sua remoção, notou-se, na mucosa gástrica, múltiplas úlceras de diversos tamanhos, alcançando até $2 \mathrm{~cm}$ de diâmetro (Figura 4). Não havia presença de alimentos na luz do órgão, nem mesmo no esôfago do animal. No intestino também foram observadas inúmeras úlceras pequenas (Figura 4), e à abertura do intestino grosso notou-se presença de conteúdo de coloração escurecida de aspecto pastoso e com presença de pelos.

O rim estava discretamente edemaciado e de coloração pálida, no entanto na região medular apresentou discreta área de coloração avermelhada. A bexiga encontrava-se repleta e à abertura notou-se sedimentos na urina. Foram coletados fragmentos do pulmão, rins, fígado e coração a fim para realização de exame histopatológico. 


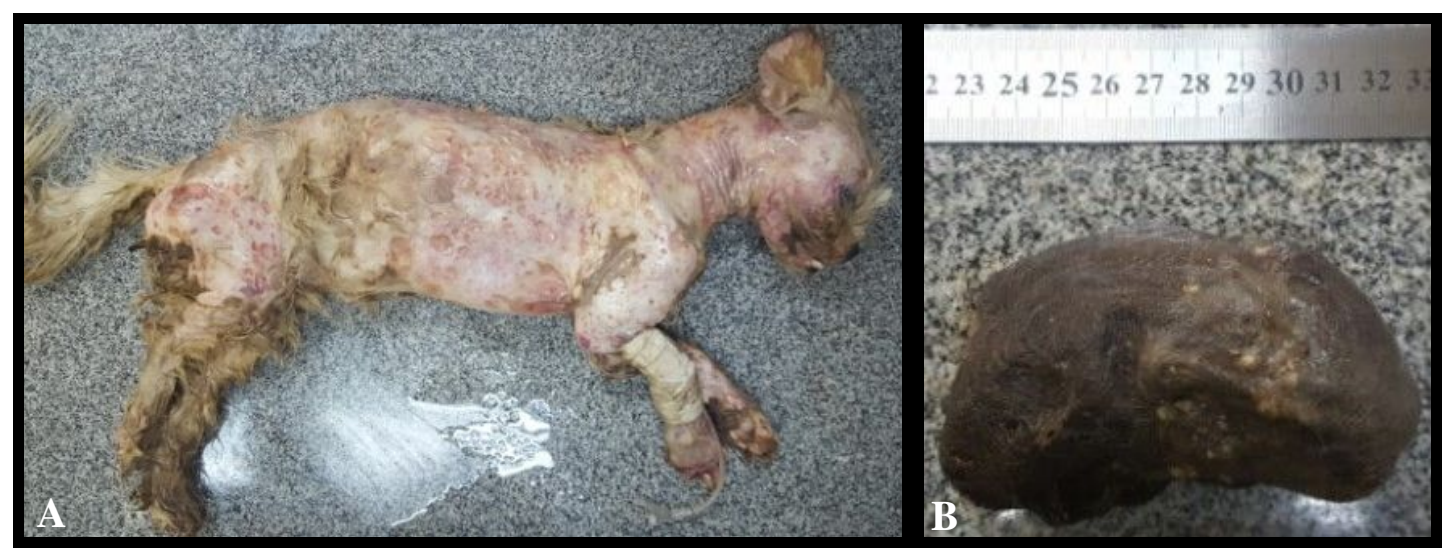

FIGURA 3 A) Cão, macho, adulto, SRD, portador de DAC, em decúbito lateral esquerdo, com descolamento de pele em praticamente toda lateral direita do corpo, com eritema e inúmeras úlceras. B) Tricobezoar de $11 \mathrm{~cm} \times 6 \mathrm{~cm}$ de tamanho, retirado do interior do estômago do animal.

Fonte: Arquivo pessoal (2016).

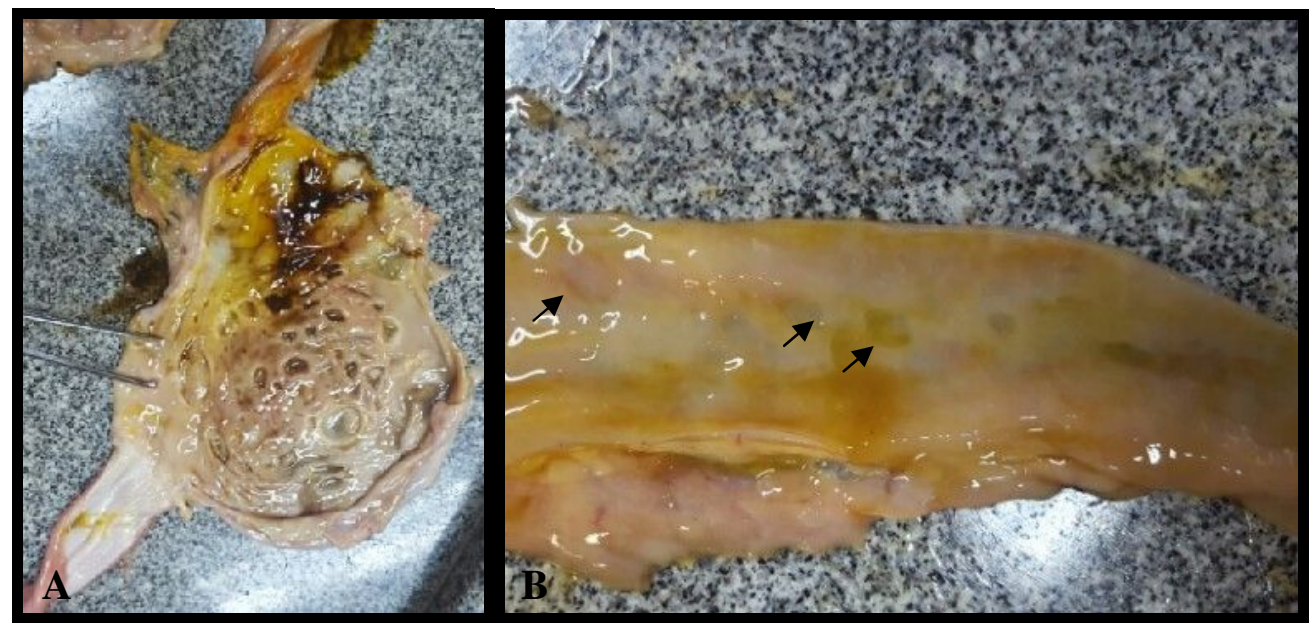

FIGURA 4 Cão, macho, adulto, SRD, portador de DAC. A) Mucosa estomacal, após retirada de tricobezoar, com inúmeras úlceras, de diversos tamanhos. B) Intestino delgado, com úlceras na mucosa (setas).

Fonte: Arquivo pessoal (2016).

Ao exame microscópico observou-se no fragmento de pulmão moderado edema intersticial e pleural, espessamento dos septos alveolares associado à presença de acentuado e difuso infiltrado inflamatório predominantemente mononuclear, com neutrófilos em menor número, além de grande quantidade de células inflamatórias na luz vascular associada à fibrina, aderida à parede, evidenciando formação de trombo. Notou-se também discretas áreas focais de enfisema.

No fígado, apesar da autólise, foi possível verificar fibroplasia periportal, discreta proliferação de ductos biliares, discreta colestase em sinusoides, moderada degeneração de hepatócitos e moderado infiltrado mononuclear difuso, 
eventualmente associado à exsudação de fibrina. Já no fragmento de coração notou-se edema intersticial discreto e, presença trombo em luz vascular.

Nos rins observou-se espessamento da membrana basal mesangial dos glomérulos, associada a hipercelularidade das células mensangiais, compatível com glomerulonefrite membrano-proliferativa, além de discreto espessamento da cápsula glomerular, acentuado infiltrado linfoplasmocitário multifocal intersticial, degeneração tubular epitelial multifocal. Em zona medular observou-se áreas multifocais de fibroplasia assim como mineralização tubular.

Os achados histopatológicos associados aos da macroscopia indicaram a existência de pneumonia intersticial crônico ativa e glomerulonefrite associadas à dermatite purulenta. Concluiu-se que o animal veio a óbito em função da presença do tricobezoar que levou a diversas ulcerações e consequente hemorragia gastrointestinal.

\section{RESULTADOS E DISCUSSÃO}

Antes de ser adotado pela tutora do presente relato, o cão havia sido encontrado abandonado na rua, e foi resgatado por uma protetora de animais. Este foi diagnosticado com dermatite atópica, podendo esta ter sido uma das causas do "descarte" do animal. Essa hipótese é reforçada por Baquero et al. (2015), os quais mencionam que a existência de doenças é o principal motivo de tutores abandonarem seus animais de estimação.

Após os cuidados necessários e posterior adoção, o cão já encontrava-se com a segunda tutora, no momento em que foi vítima de maus tratos. O cão deste estudo demandava atenção especial bem como tratamento permanente, devido à dermatite atópica canina, sendo a necessidade desta conduta igualmente mencionada por Medeiros (2017). Mendonça e Melo (2014), ainda ressaltam a importância da guarda responsável, na qual o tutor se responsabiliza em atender às necessidades básicas do animal, garantindo seu bem-estar, fato que no presente relato foi totalmente negligenciado.

Diogo et al., (2014) mencionam o elevado impacto que a doença ocasiona na qualidade de vida do cão e de seu tutor. Cita ainda que o diagnóstico precoce e o tratamento adequado da enfermidade são essenciais para minimizar os efeitos causados pela DAC na qualidade de vida dos cães e seus tutores. Além disso, por ser uma enfermidade que necessita de tratamento contínuo. Estes mesmos autores enfatizam a importância da compreensão, sobre a doença, por parte do tutor, sendo esta essencial para que haja efetividade do tratamento, evitando assim que a enfermidade evolua. No entanto, no caso em questão, uma das justificativas da tutora para o não encaminhamento do animal ao atendimento médico veterinário prévio, estava relacionada a fatores financeiros, fato que levou ao agravamento da situação. De acordo com Mendonça e Melo (2014), pesquisas apontam que apesar do nível socioeconômico não estar relacionado com o êxito da adoção, por vezes é indicado, pelos tutores, como fator complicador da manutenção do animal.

No presente estudo, a DAC evoluiu para uma dermatite purulenta, acometendo extensas áreas da pele, o que possivelmente decorreu da ausência de tratamento adequado, fator determinante para o agravamento da situação clínica. Diogo et al., (2014), Medeiros (2017) e Timm et al., (2018) afirmam que, nesta enfermidade, é comum a existência de infecções secundárias, que por vezes são 
bacterianas, levando assim ao desenvolvimento de piodermites (MATOS et al, 2012), ocasionando maior comprometimento do estado geral do animal.

Segundo Horta e Costa Val (2013), as dermatites normalmente geram prurido e queda de pelo, portanto pressupõe-se, neste relato, que o intenso prurido causado pela DAC, associado à alopecia, tenha possibilitado à ingestão de pelos, com posterior formação de tricobezoar gástrico. Albernaz et al. (2017) relatam que a formação de tricobezoar está associada a tricofagia, principalmente em seres humanos.

Além dos sinais clínicos descritos no presente caso, Couceiro et al. (2016), Finale et al., (2018) e Khan et al., (2018), ainda relatam outras complicações comuns como perfuração, obstrução e sinais inespecíficos como dor abdominal, náuseas, vômito, hematêmese, anorexia, anemia, melena e halitose. Portanto o tamanho do corpo estranho, no interior do estômago, do cão deste estudo, impossibilitava a ingestão de alimentos e dificultava o consumo de água, condição que ocasionou em desnutrição e desidratação. A presença do tricobezoar levou a irritação da mucosa e formação de múltiplas úlceras que culminaram em hemorragia, choque hipovolêmico e óbito do animal.

Como a tutora do animal, no momento em que este foi resgatado para ser encaminhado ao médico veterinário, não relatou, em momento algum, sinais clínicos que não os de pele e a apatia, não se suspeitou da presença de corpo estranho no sistema digestivo do cão. Sendo a primeira suspeita, septicemia decorrente da infecção purulenta generalizada na pele.

Portanto, o descaso da tutora impediu o diagnóstico correto, demonstrando que não somente a falta de conhecimento e a dificuldade financeira foram fatores determinantes neste caso, mas principalmente o abandono do animal em relação aos cuidados básicos necessários, por parte da responsável, para que não chegasse nesta situação, uma vez que a mesma também poderia ter entrado em contato com a pessoa de quem adotou o cão relatando sua situação. Fauth (2014) menciona em seu artigo, o decreto de lei 24.645/34, artigo 3ํ de junho de 1934, o qual deixa claro que a falta de assistência médico veterinária, bem como abandono de um animal doente, são consideradas situações de maus tratos.

\section{CONCLUSÕES}

Situações de maus tratos, decorrentes de negligência, por falta de assistência veterinária no momento certo e ausência de tratamento adequado aos cães, ainda são uma realidade. A falta de cultura e dificuldades financeiras são fatores importantes, mas o descaso em relação ao animal doente ainda é o maior problema. Desfechos como o do presente caso são intoleráveis e por isso são considerados crimes. Além disso, foi possível concluir que a falta de tratamento correto de DAC pode ocasionar na morte do animal, devido aos diversos problemas secundários decorrentes desta enfermidade.

\section{REFERÊNCIAS}

ALBERNAZ, V. G. P.; CONCEIÇÃO, R.T.; EISING, T.C.; FABRIS, I.A.; MAMPRIM, M.J.; et al, Partial Obstruction of the Small Intestine by a Trichobezoar in a Dog. Acta Scientiae Veterinariae. v. 45, n. 1, art. 210, 2017. Disponível em: <http://www.ufrgs.br/actavet/45-suple-1/CR_210.pdf> https://doi.org/10.22456/1679-9216.85862 
ALVES, B. H.; VIANA, J. A.; LEIRA, M. H.; RODRIGUES, N. do P. A.; PRISTO, A. L. de $P$., et al, Dermatite atópica canina: Estudo de caso. Publicações em Medicina Veterinária e Zootecnia. v. 12, n. 8, p. 1-6, 2018. Disponível em: < http://www.pubvet.com.br/uploads/e8a84bff9879048d7ffd64057c59717a.pdf> doi: https://doi.org/10.31533/pubvet.v12n8a154.1-6

BAQUERO, O. S.; CHIOZZOTTO, E. N.; GARCIA, R. de C. M.; AMAKU, M.; FERREIRA, F. Demographic characteristics of owned of dogs and cats of Votorantim, São Paulo. Ciência Rural, v. 45, n. 11, p. 2039-2043, 2015. Disponível em: $\quad<h t t p: / / w w w . s c i e l o . b r / s c i e l o . p h p ? s c r i p t=s c i=a r t t e x t ; p i d=S 0103$ 84782015001102039> doi: http://dx.doi.org/10.1590/0103-8478cr20141646

BIZIKOVA, P.; SANTORO, D.; MARSELLA, R.; NUTTALL, T.; EISENSCHENK, M. N. C., et al, Review: Clinical and histological manifestations of canine atopic dermatitis. Veterinary Dermatology. v. 26, n. 2, p. 79-84, 2015. Disponível em: < https://onlinelibrary.wiley.com/doi/full/10.1111/vde.12196> doi: https://doi.org/10.1111/vde.12196

COUCEIRO, A.; VIVEIRO, C.; CAPELÃO, G.; NOBREA, J.; LAUREANO, M. et al Trichobezoar --- A Rare Cause of Abdominal Mass and Gastric Outlet Obstruction. GE-Portuguese Journal of Gastroenterology. v. 23, n. 1, p. 50-53, 2016. Disponível em: https://www.sciencedirect.com/science/article/pii/S2341454515001118> doi: https://doi.org/10.1016/j.jpge.2015.08.003

DIOGO, J. E.; LIMA, S. R.; GASPARETTO, N. D.; ALMEIDA, A. B. P. F.; PESCADOR, A. C. et al, Estudo retrospectivo de biópsias de pele: um comparativo da dermatite atópica canina com as demais dermatoses. Acta Veterinaria Brasilica. v. 8 , n. 4 , p. 286-289, 2014. Disponível em: < https://periodicos.ufersa.edu.br/index.php/acta/article/view/4634 > doi: https://doi.org/10.21708/avb.2014.8.4.4634

DOMINGUES, L. R.; CESAR, J. A.; FASSA, A. C. G.; DOMINGUES, M. R. Guarda responsável de animais de estimação na área urbana do município de Pelotas, RS, Brasil. Ciência e saúde coletiva. v. 20, n. 1, p. 185-192, 2015. Disponível em: < http://www.scielo.br/pdf/csc/v20n1/1413-8123-csc-20-01-00185.pdf > doi: 10.1590/1413-81232014201.19632013

FAUTH, J. de A. Medidas protetivas: a defesa do animal doméstico. Cognitio Juris, n. $10, \quad$ p. 121-141, 2014. Disponível em: < http://www.ambitojuridico.com.br/site/index.php?n_link=revista_artigos_leitura;artigo_ id $=13742>$

FINALE, E.; FRANCESCHINI, P.; DANESINO, C.; BARBAGLIA, M.; GUALA, A. Rapunzel syndrome: how to orient the diagnosis. Pediatric reports. v. 10, n. 2, p. 37-38, 2018. Disponível em: < https://www.researchgate.net/publication/326373915_Rapunzel_syndrome_How_to_ orient_the_diagnosis > doi: $10.4081 /$ pr.2018.7689 
GROSSEL, L. A.; POVALUK, M. Medidas profiláticas para amenizar a questão das zoonoses ocasionadas por cães abandonados nas ruas do bairro faxinal, Mafra - SC Saúde e Meio Ambiente v. 5, n. 2, p. 3-20, 2016. Disponível em: < http://www.periodicos.unc.br/index.php/sma/article/view/888> doi: https://doi.org/10.24302/sma.v5i2.888

HORTA, R. dos S.; COSTA VAL, A. P. da Exames complementares no diagnóstico dermatológico em pequenos animais. Cadernos técnicos de medicina veterinária e zootecnia: dermatologia em cães e gatos, n. 71, p. 23-31, 2013. Disponível em: $<$

https://issuu.com/escoladeveterinariaufmg/docs/caderno_tecnico_71_dermatologia_c ae>.

KHAN S.; KHAN, I-A.; ULLAH, K.; KHAN, S.; WANG, X., et al, Etiological aspects of intragastric bezoars and its associations to the gastric function implications: A case report and a literature review. Medicine (Baltimore). v. 97, n. 27, e11320, 2018. Disponível em:

https://www.researchgate.net/publication/326179425_Etiological_aspects_of_intraga stric_bezoars_and_its_associations_to_the_gastric_function_implications_A_case_r eport_and_a_literature_review > doi: 10.1097/MD.0000000000011320

MACPHAIL, C. Gastrointestinal Obstruction. Clinical Techniques in Small Animal Practice, v. 17, n. 4, p. 178-183, 2002. Disponível em: < https://www.sciencedirect.com/science/article/pii/S1096286702800494> doi: https://doi.org/10.1053/svms.2002.36606

MATOS, C. B.; MADRID, I.M.; SANTIN, R.; AZAMBUJA, R.H.; SCHUCH, I.; MEIRELES, M.C.A., et al, Dermatite multifatorial em um canino. Arquivo Brasileiro de Medicina Veterinária e Zootecnia. v. 64, n. 6, p. 1478-1482, 2012. Disponível em: $\quad<\quad$ http://www.scielo.br/scielo.php?script=sci_abstract;pid=S010209352012000600011;lng=p;tlng=pt> doi: http://dx.doi.org/10.1590/S010209352012000600011

MEDEIROS, V. B Dermatite atópica canina. Journal of Surgery and Clinical Research, v. 8, n. 4, p. 106-117, 2017. Disponível em: < https://www.researchgate.net/publication/320543098_Dermatite_atopica_canina> doi: $10.20398 /$ jscr.v8i1.13044

MENDONÇA, T. R. O de; MELO, M. de F. A. de Q. Adoção de Cães por Universitários: um estudo ator-rede sobre a relação humano/não-humano. Pesquisas e Práticas Psicossociais, v. 9, n. 1, p. 130-141, 2014. Disponível em: < http://www.seer.ufsj.edu.br/index.php/revista_ppp/article/view/833>

PÉREZ, B. G.; CRESPO, J. J. M.; BELMONTE, D. G. Tricobezoar como causa de obstruccíon intestinal em mujer adolescente. Gastroenterología y Hepatología. v.37, n.9, p.545-546, 2014. Disponível em: < https://www.researchgate.net/publication/264624960_Tricobezoar_como_causa_de_ 
obstruccion_intestinal_en_mujer_adolescente

doi: 10.1016/j.gastrohep.2014.02.002

PUCHEU-HASTON, C. M. Atopic dermatitis in the domestic dog. Clinics in Dermatology. v. 34, n. 2, p. 299-303, 2016. Disponível em: < https://www.sciencedirect.com/science/article/pii/S0738081X15001893> doi: https://doi.org/10.1016/j.clindermatol.2015.10.010

NOLI, C.; COLOMBO, S.; CORNEGLIANI, L.; GHIBAUDO, G.; PERSICO, P.; VERCELLI, A.; GALZERANO, M. Quality of life of dogs with skin disease ando $f$ their owners. Part 2: administration of a questionnaire in various skin diseases and correlation to efficacy of therapy. Veterinary Dermatology, v.22, n.4, 2011. Disponível em:< https://onlinelibrary.wiley.com/doi/full/10.1111/j.13653164.2011.00956.x> doi: https://doi.org/10.1111/j.1365-3164.2011.00956.x

SANTOS, M. G. O.; SANTOS, M. M. dos Perfil da dermatite atópica canina em Salvador - Brasil. Publicações em Medicina Veterinária e Zootecnia. v. 10, n. 11, p. $839 \quad-\quad 843, \quad 2016.2$ Disponível em: < http://www.pubvet.com.br/uploads/f152dd783a5fd5f6923547b225bc95a1.pdf> doi: http://doi.org/10.22256/pubvet.v10n11.839-843

SARIDOMICHELAKIS, M. N.; OLIVRY, T. An update on the treatment of canine atopic dermatitis. The veterinary journal. v. 207, p. 29-37, 2016. Disponível em: < https://www.ncbi.nlm.nih.gov/pubmed/26586215 > doi: 10.1016/j.tvjl.2015.09.016

TIMM, K.; MUELLER, R. S.; NETT-METTLER, C. S. Long-term effects of intralymphatic immunotherapy (ILIT) on canine atopic dermatitis. Veterinary Dermatology. v. 29, n. 2, p. 123-127, 2018. Disponível em: < https://onlinelibrary.wiley.com/doi/full/10.1111/vde.12517> doi: https://doi.org/10.1111/vde.12517 